\title{
El caso de las normas contables globales: argumentos y evidencias ${ }^{1}$
}

\author{
Ann Tarca $^{2}$
}

Recibido: 10 de agosto de 2015

Aprobado: 15 de noviembre de 2015

\section{Tarca, A. (2015). El caso de las normas contables globales: argu- mentos y evidencias. Activos, 25, 75-106}

\section{Clasificación JEL: G18, G38, G14.}

\section{Resumen}

Este artículo resume los argumentos a favor de un conjunto común de normas de contabilidad y las fuerzas que han promovido la adopción de las Normas Internacionales de Información Financiera -en adelante NIIF-. El uso generalizado de las NIIF a partir de 2005 ofrece una oportunidad para la investigación empírica sobre sus beneficios. Además, se resumen los resultados de los estudios que son relevantes para evaluar el papel de las NIIF en los mercados de capitales desarrollados y emergentes.

1 Agradecemos al profesor Carlos Rico Bonilla de la Facultad de Contaduría Pública de la Universidad Santo Tomás la colaboración en las labores de autorización para la publicación de este documento, así como en la tarea de su traducción. Este fue publicado originalmente como documento de reflexión en la página de la fundación IFRS en el año 2012. La Revista Activos lo reproduce teniendo en cuenta la importancia que sus aportes pueden hacer para el debate sobre la convergencia al modelo normativo IFRS que se adelanta actualmente en Colombia.

2 Catedrática de contabilidad - University of Western Australia. Colaboradora académica - Investigación, Fundación IFRS. Las opiniones expresadas en este artículo son de la autora, y no comprometen la posición del IASB o de sus miembros. 


\section{Palabras clave}

Normas Internacionales de Información Financiera, mercados de capitales, investigación empírica, análisis de impacto.

Tarca, A. (2015). The case of global accounting standards: arguments and evidence. Activos, 25, 75-106

\section{Summary}

This article summarizes arguments in favor of a common set of accounting standards and the forces that have promoted the adoption of International Financial Reporting Standard - known as IFRS - The widespread use of IFRS since 2005 provides an opportunity for empirical research on its benefits. In addition, there is a summary of the results of some studies that are relevant to evaluate the role of IFRS for developed and emerging capital markets.

\section{Keywords}

International Financial Reporting Standard, capital markets, empirical research, impact analysis.

Tarca, A. (2015). Le cas des normes comptables globales : arguments et évidences. Activos, 25, 75-106

\section{Résumé}

Cet article résume les arguments en faveur d'un ensemble de normes comptables et les forces qui ont favorisée l'adoption des normes internationales d'information financière - NIIF. L'utilisation généralisée des NIIF à partir du 2005 offre une opportunité pour l'investigation empirique sur les avantages. 
En outre, les résultats des études qui sont pertinentes pour évaluer le rôle des NIIF sur les marchés de capitaux développés et émergents sont résumés.

\section{Mots clés}

Normes internationales d'information financière, marché de capitaux, investigation empirique, analyse d'impact.

\section{Introducción}

Los beneficios esperados de las normas contables globales son convincentes. El uso de un conjunto de normas de alta calidad por empresas en todo el mundo, tiene el potencial de optimizar la comparabilidad y transparencia de la información financiera y reducir los costos de preparación de los estados financieros. Cuando las normas son aplicadas de forma rigurosa y coherente, los participantes de los mercados de capitales tienen información de mayor calidad y pueden tomar mejores decisiones. En consecuencia, se asignan fondos más eficientemente y las empresas pueden lograr un menor costo de capital.

Estos argumentos han sido utilizados para apoyar la adopción de las NIIF en los reportes financieros de las entidades consolidadas que cotizan en las bolsas de valores en los Estados miembros (CE1606/2002) de la Unión Europea (UE). Otros países han citado razones similares para adoptar las NIIF (véase Brown, 2011), lo que refleja la demanda de normas de alta calidad que puedan mejorar la excelencia y la comparabilidad de la información financiera y promover el desarrollo y la integración internacional de los mercados de capital nacionales.

Por primera vez en la historia, tenemos un número sustancial de firmas domiciliadas en diferentes países que usan normas comunes. En consecuencia, se pueden reunir pruebas sobre el grado en que se observan beneficios en los mercados de capitales. Este artículo presenta resultados de 
investigaciones sobre el impacto de la adopción de las NIIF desde el 2005, cuando se convirtieron en las normas contables legalmente requeridas para más de 6.000 empresas de la UE y para corporaciones de otras naciones, incluyendo Australia y Sudáfrica.

La siguiente sección resume la evidencia sobre el impacto de las NIIF en las operaciones eficientes de los mercados de capital, teniendo en cuenta datos relacionados con los precios de las acciones y los beneficios, además con las actividades de los inversionistas internacionales y los analistas financieros.

En las siguientes secciones se considera el impacto de las NIIF en los países en vías de desarrollo y en aquellos en los que los mercados de capital son maduros y presentan evidencia relevante para evaluar el papel de las NIIF, tanto antes como después de la crisis financiera de 2007-2008. En la sección final se concluye el artículo.

\section{La adopción de las NIIF y la eficiencia del mercado}

Una pregunta fundamental es si las NIIF han modificado la información disponible para los participantes del mercado en una dirección provechosa, esto es, ¿̇son estos más eficientes cuando se usan las normas internacionales? Se espera que la información bajo NIIF suministrada por las firmas a los participantes del mercado difiera significativamente de la basada en los Principios de Contabilidad Generalmente Aceptados (PGCA) nacionales, debido a las divergencias entre los requisitos de las normas nacionales y de las NIIF. La medida en que el cambio hacia las NIIF proporciona datos más relevantes que se traducen en beneficios observables en los mercados de capital, es una cuestión que se está abordado en la investigación actualmente.

Algunos estudios reúnen pruebas sobre los cambios en la liquidez del mercado y el costo del capital de las firmas, como una fórmula de medición del impacto de las NIIF. En uno de los primeros trabajos a gran escala sobre 
empresas que adoptaron las NIIF de forma obligatoria, Daske et al., (2008) concluyen que se presentó aumento de la liquidez del mercado tras la introducción de las NIIF. Además, encontraron evidencia de una disminución del costo de capital en las empresas y un aumento del valor patrimonial que ocurrió antes de la fecha oficial de adopción. En una investigación relacionada, los autores hallaron que es más probable que beneficios tales como una mejor liquidez y un menor coste de capital se produzcan en corporaciones "serias" en su proceso de adopción de las NIIF (definidas como firmas con un compromiso con la transparencia) (Daske et al., 2011).

Li (2010) encontró que entidades obligadas a aplicar las NIIF se benefician de una reducción de sus costos de capital de forma inmediata en el periodo de adopción, lo que refleja incrementos en la información a revelar y la comparabilidad de la información mejorada. Sin embargo, la reducción únicamente se producía en países con un nivel fuerte de enforcement legal ${ }^{3}$.

Palea (2007) considera los costos de los efectos del capital en las instituciones financieras. Reporta menores costos de capital para instituciones financieras de la UE que utilizan las NIIF, en comparación con otras que aplican normas nacionales y la Cuarta y Séptima Directivas, un resultado que es consistente con los objetivos de adopción de las NIIF de la Comisión Europea (CE 1606/2002).

Los investigadores han empleado durante mucho tiempo los precios de las acciones para mostrar información acerca de la utilidad de los datos financieros (Ball y Brown, 1968; Beaver, 1968). En esta tradición, Beuselinck et al., (2009) analizan la synchronicity ${ }^{4}$ de la rentabilidad de las acciones para aquellos que adoptaron de forma obligatoria las NIIF en la UE. Llegan a la conclusión de que la adopción de las NIIF revela nueva información

3 Los investigadores utilizan el término enforcement para referirse a la infraestructura legal nacional en la que se desenvuelve la información financiera, es decir, los sistemas jurídicos y de gobierno que protegen a los inversionistas y acreedores y disciplinan a quienes llevan a cabo actividades ilegales.

4 Synchronicity se refiere a la medida en que los valores de las acciones individuales se mueven en relación con los precios del mercado en general. 
específica de la empresa, que posteriormente reduce la sorpresa de información a revelar futura.

Landsman et al., (2011) consideran el impacto del uso de las NIIF sobre los precios de las acciones y su negociación (volatilidad del rendimiento anormal y volumen de transacciones). Concluyen que la información contenida en los anuncios de ganancias ha mejorado para las empresas que usan las NIIF al reducirse el retraso en la presentación de reportes, aumentar el análisis de los datos y la inversión extranjera. También encuentran que el efecto de las NIIF depende del nivel de enforcement en un país.

Otros estudios exploran el impacto de las NIIF en los participantes del mercado. En relación con los inversionistas, las investigaciones suministran pruebas de varios beneficios que pueden estar vinculados con la aplicación por parte de las firmas de normas comparables y de alta calidad. Brüggemann et al., (2009) examinan las inversiones en empresas extranjeras realizadas por inversores particulares en el mercado abierto de la Bolsa de Valores de Frankfurt (4.869 corporaciones de 31 países). Reportan un aumento en la actividad comercial tras la adopción de las NIIF. Así, los investigadores concluyen que las NIIF tienen el potencial de promover mayores inversiones en capital extranjero por parte de inversores particulares.

DeFond et al., (2011) también investigan la relación entre las NIIF y las inversiones transfronterizas. Encuentran que la posesión en fondos mutuos de inversión estadounidenses aumenta en el caso de aquellos inversores que adoptan de forma obligatoria, pero solo cuando la adopción es vista como "creíble". En consecuencia, del mismo modo que Daske et al., (2009), señalan los beneficios de las NIIF que surgen gracias a mejoras de la comparabilidad, pero advierten que la forma en que las normas se aplican (es decir, la manera en que son usadas por las empresas) es de crucial importancia para la obtención de los beneficios. Shima y Gordon (2011) muestran que tanto las normas como el enforcement son necesarios para aumentar la inversión extranjera. 
Yu (2010) también examina la participación en fondos mutuos de inversión. Concluye que la adopción de las NIIF aumenta las transferencias transfronterizas al reducir los costos de procesamiento de información de los inversionistas extranjeros, a través de la mejora en la comparabilidad de la información financiera e indirectamente mediante la reducción de otras barreras como la distancia geográfica. Su evidencia también sugiere que la armonización de diferentes regímenes de presentación de informes contables es una manera más eficaz para atraer capitales foráneos que la simple mejora de los regímenes de cada país.

Francis et al., (2012) advierten un mayor volumen de las actividades de fusiones y adquisiciones, con primas más elevadas, cuando los países tienen PCGA parecidos. También encuentran más de estas transacciones en naciones que adoptaron las NIIF en el 2005, una tendencia más pronunciada en países que tenían una baja similitud entre sus PCGA y las NIIF antes de la adopción. Los investigadores concluyen que PCGA análogos reducen los costos de información, lo que aumenta la competencia entre los compradores y permite mayores ganancias para los accionistas de destino.

Flourou y Pope (2011) exploran la cuestión de si la obligatoriedad de las NIIF induce un aumento en la propiedad institucional. Teniendo en cuenta el año de adopción y uno posterior, reportan incrementos de la propiedad y, en consecuencia, muestran que las NIIF afectan las decisiones de asignación de los inversores institucionales. Los cambios de propiedad relacionados con las NIIF son más altos para los inversores de valor y crecimiento, que son más propensos a hacer uso de los estados financieros en sus decisiones inversoras. Una advertencia importante en este estudio es que los efectos positivos de las NIIF solo se encuentran en los países con un nivel de enforcement legal estricto y bajos niveles de corrupción. Este punto se tratará más adelante en el artículo.

Muchos estudios indagan por los efectos del uso obligatorio de las NIIF para los analistas del mercado, ya que son importantes usuarios de los estados financieros. Las investigaciones sobre las NIIF se basan en una literatura preexistente que sugiere que una información de los estados financieros de 
alta calidad facilita la labor de estos agentes (Lang y Lundholm, 1996; Hope, 2003). En consecuencia, se espera que ellos se favorezcan si las normas internacionales optimizan la pertinencia, transparencia y comparabilidad de la información. Un estudio de Bae et al., (2008) sobre analistas que siguen firmas extranjeras sugiere que estos se benefician cuando las normas contables son más similares. Específicamente, cuando los PCGA de un país están más cerca de las normas internacionales (NIC en su estudio), los analistas extranjeros son más propensos a seguir y ofrecer pronósticos más precisos para las empresas de ese país.

Varios estudios realizados en el periodo en que las NIIF se hacen obligatorias concluyen que la adopción ha beneficiado a los analistas financieros. Horton et al., (2013) encuentran que los analistas que siguen empresas que aplican las NIIF tienen una mayor precisión en sus previsiones en comparación con aquellos que están pendientes de firmas que no toman estas normas. Concluyen que las NIIF han mejorado el entorno de la información mediante el aumento de la calidad y la comparabilidad de la misma. Otros investigadores también observan mejoras en la exactitud de los pronósticos basados en estudios sobre compañías de la UE (Jiao et al., 2012) y de determinados países, tanto de economías de mercado desarrolladas como en vías de desarrollo (Panaretou et al., 2013; Choi et al., 2010; Ernstberger et al., 2008; Hodgdson et al., 2008; Cotter et al., 2012). Beuselinck et al., (2010) hallan que las NIIF favorecen la capacidad de los analistas para incorporar datos específicos de la firma y la industria en el precio de las acciones, y reducen la ventaja de información privada que está en manos de los inversionistas institucionales. En otro estudio de empresas que aplican las NIIF, Beuselinck et al., (2009) señalan que la precisión de la información aumentó después del 2006, pero el consenso entre los analistas no cambió, lo que sugiere un incremento de la exactitud tanto de la información general como de la privada. Los analistas que siguen compañías en más de un país experimentaron el mayor beneficio posterior a la aplicación de las NIIF en términos de la precisión de la información privada. 
Byard et al., (2011) notaron beneficios para los analistas financieros en el periodo inmediato de post-adopción de las NIIF en las compañías de la UE. Las ventajas se observan en los países en que los PCGA nacionales y las NIIF tienen las mayores diferencias y además hay instituciones fuertes que dan su apoyo a la adopción, es decir, donde el sistema jurídico es sólido y ofrece protecciones a los inversionistas. Tan et al., (2011) señalan que la aplicación obligatoria de las NIIF atrae a los analistas extranjeros a una empresa y también mejora la precisión de los pronósticos. El aumento de los análisis se asocia positivamente con el grado de diferencia entre los PCGA locales y las NIIF y la medida en que la adopción elimina esta brecha. La adopción de las NIIF además atrae a más analistas financieros locales, aunque no influye significativamente en su precisión de los pronósticos.

La mayoría de las investigaciones se centran en los efectos de las NIIF relacionados con la negociación (trading) en los mercados de valores. Sin embargo, Beneish et al., (2010) muestran que las NIIF tienen un mayor impacto en los mercados de deuda que en los de valores. Los autores observan que los incrementos en la inversión de capital extranjero en el periodo posterior a la adopción se limitan a los países con alta calidad del gobierno corporativo (o que está en procesos de mejora). No obstante, el aumento en los flujos de las inversiones en deuda exterior no depende de la calidad del gobierno corporativo, de conformidad con las cláusulas en los contratos de bonos que se utilizan para compensar las deficiencias nacionales en la protección del inversor. El aumento de las inversiones deriva tanto de los Estados Unidos como de otros países no adoptantes, lo que sugiere que dicho aumento está vinculado a la calidad de las NIIF.

Los resultados de estos estudios suministran evidencia de que la adopción generalizada de las NIIF ha traído beneficios a los mercados de capitales. Los investigadores usualmente concluyen que los mismos derivan de la naturaleza de las normas (en comparación con los PCGA anteriores) y su uso por empresas de diferentes países, lo que favorece la comparabilidad de la información para los inversionistas y los analistas financieros internacionales. 
Un hilo común en la literatura académica es que los beneficios de las normas estandarizadas comunes no pueden ser alcanzados solo por estas. Dicha cuestión se planteó antes de la adopción generalizada de las NIIF. Ball et al., $(2000,2003)$ ponen de relieve la importancia de los incentivos en la determinación de cómo se utilizan las normas en la práctica. El punto también ha sido abordado por los investigadores en NIIF, en particular Christensen et al., (2008), quienes argumentan que incentivos firmes son más importantes que las normas contables en sí mismas en la comprensión de los efectos de las NIIF.

Utilizando datos relativos al periodo posterior a la adopción de 2005, muchos investigadores han concluido que el escenario en el que tiene lugar la presentación de los reportes bajo las NIIF tiene una fuerte influencia a la hora de determinar que los beneficios potenciales de las NIIF se puedan capitalizar (Daske et al., 2008; Beuselink et al., 2009; Flourou y Pope, 2011; Landsman et al., 2011; Christensen et al., 2010, 2012). El éxito de las normas comunes no depende sólo de la calidad de las normas emitidas por el IASB. Críticamente, el éxito requiere también de una infraestructura para apoyar que las NIIF estén en su lugar a nivel nacional e internacional.

En consecuencia, no todos los estudios reportan mejoras cuando las empresas adoptan las NIIF. Como se señaló anteriormente, los beneficios están estrechamente vinculados con el grado en que los anteriores PCGA nacionales y las NIIF difieren y el nivel de enforcement (o, dicho de otra manera, el marco legal y de negocios y la cultura de información financiera) de los distintos países. Los investigadores que se ocupan de los incentivos de los gerentes argumentan que las ventajas de las NIIF solo surgirán si las empresas tienen los estímulos para proporcionar informes de alta calidad, ya sea debido a circunstancias individuales o en respuesta a los requisitos legales de información y de gobierno corporativo en los países en los que operan y los mercados donde están listados en bolsa.

Wang y Yu (2009) encuentran que mejores normas de contabilidad (por ejemplo, las NIIF) tienen efectos positivos para el contenido informativo de los precios de las acciones en los países cuyo sistema jurídico tiene su 
origen en el derecho común, con una mejor protección de los accionistas y un enforcement legal más estricto. Estos estudios hacen énfasis en un punto señalado por muchos investigadores: el entorno institucional afectará la medida en que los beneficios y las NIIF se pueden realizar.

No toda la investigación apoya la afirmación de que las NIIF son provechosas para los mercados de capital. Algunos investigadores cuestionan si la comparabilidad mejora tras la adopción de las NIIF, e indican la evidencia de la continuación de opciones de política pre-adopción (Kvaal y Nobes, 2010, 2011).

Lang et al., (2010) miden el movimiento conjunto de las ganancias (sustentado en la covarianza de resultados entre empresas de diferentes países) y la comparabilidad contable (basada en la relación entre los resultados y la rentabilidad de una firma, comparando entre firmas de varias naciones). Encuentran que mientras se amplía el movimiento conjunto de las empresas que aplican las NIIF, la comparabilidad no lo hace. Además, el incremento del movimiento conjunto se asocia con un entorno de información más pobre (medido como una disminución en la cobertura del análisis, la precisión de los pronósticos y un aumento del diferencial de precios comprador-vendedor).

\section{Las NIIF y el desarrollo económico}

Los estudios sobre el desarrollo del mercado de capital muestran fuertes vínculos entre la infraestructura legal y financiera de una nación y la madurez de su mercado de capital. En un estudio de 49 países con datos de 1994, La Porta et al., (1997) muestran que los estados con la protección del inversor más precaria (medida tanto por el carácter de las reglas jurídicas y la calidad del enforcement) tienen mercados de capitales más pequeños y estrechos. En un trabajo relacionado, La Porta et al., (1998) concluyen que los países de derecho común cuentan con la más fuerte protección jurídica de los inversores, mientras que en las naciones que operan con derecho civil francés dicha protección es la más débil. 
Los países de derecho civil alemán y escandinavo están en un lugar intermedio. Jackson y Roe (2009) además exploran la relación entre la actividad legal y el desarrollo del mercado de capitales. Ellos argumentan que enforcement público y privado (normalmente asociados con los sistemas del derecho civil y del derecho común, respectivamente) son importantes para el desarrollo de los mercados, resaltando la práctica de algunos países que aplican el derecho común para hacer uso de mecanismos que normalmente se observan en las jurisdicciones civiles.

Otros estudios apuntan a la interrelación del marco institucional y la calidad de la información contable. Por ejemplo, Leuz et al., (2003) concluyen que el entorno legal e institucional de un país influye en las características de los resultados reportados. Encuentran que las economías abiertas (con mercados de valores grandes, una concentración más dispersa de propiedad, derechos fuertes para los inversores, y fuerte enforcement legal) cuentan con mercados de valores más grandes, una concentración más baja de la propiedad, derechos más amplios para los extranjeros, una mayor revelación y una fuerte aplicación legal en comparación con las economías cerradas. La información financiera y su revelación se considera una parte importante de la estructura institucional que afecta a la transparencia (Bushman et al., 2004).

Frost et al., (2006) hallan que la fuerza del sistema de revelación de una bolsa de valores (revelaciones, monitoreo y enforcement de la información financiera) se asocia positivamente con el desarrollo del mercado, después de controlar variables como el sistema legal, la protección jurídica de los inversores, el tamaño del mercado y otros aspectos relevantes.

Dada la importancia del marco institucional para el desarrollo del mercado, no es de extrañar que las iniciativas para promover el crecimiento del mercado de capitales impliquen el desarrollo de una sólida infraestructura financiera, incluyendo la utilización de normas contables de alta calidad. El Banco Mundial y el FMI han fomentado el uso de las NIIF para apoyar el crecimiento de los mercados de capital y, por esta vía, la estabilidad y el avance económico mundial (Hegarty et al., 2004). 
La observancia de normas y códigos (ROSC, por sus siglas en inglés) del Banco Mundial (2011) proporciona muchos ejemplos de países que adoptan las NIIF para mejorar la calidad de la información financiera de las empresas privadas y las del sector público, para promover el desarrollo de conocimientos técnicos de contabilidad dentro del país y para atraer capital extranjero.

Hope et al., (2006) examinan 38 países que han adoptado voluntariamente las NIIF antes de finalizar el año 2004. Concluyen que las normas son un vehículo a través del cual los países pueden mejorar la protección de los inversionistas y hacer mercados de capital más accesibles para los inversores extranjeros. Posteriormente Gordon et al., (2012) muestran que los flujos de inversión extranjera directa aumentan en los países en desarrollo que adoptan las NIIF. Estudian las empresas de 124 países en el periodo 1996-2009 y observan un beneficio directo para las economías en desarrollo (pero no para las ya desarrolladas), proporcionando pruebas de que el estímulo del Banco Mundial sobre el uso de las NIIF es ventajoso.

Los países que utilizan las NIIF incluyen a Corea del Sur, donde las normas plenas se hicieron obligatorias a partir de 2011 en un intento por mejorar la transparencia y la percepción internacional sobre la cultura de las empresas, con el fin de atraer más inversión foránea (Fifield, 2007).

En Malasia se aplican las NIIF desde 2012. Allí el presidente del Malaysian Accounting Standards Board declaró que la adopción no se trataba solo de "seguir la corriente", sino de reconocer que las empresas malasias operan en un entorno global. Las NIIF permiten que las empresas y los mercados del país sean reconocidos internacionalmente (MASB, 2008). Malasia previamente había armonizado parcialmente las normas nacionales con las NIIF. En una revisión de los informes financieros según los PCGA nacionales y las normas armonizadas, Wan Ismail et al., (2010) informan de una mayor calidad del resultado, mientras que Kadri y Zulkifli (2008) encuentran mayor relevancia del valor de los números contables para las empresas de Malasia en virtud de las normas (armonizadas) revisadas. La 
investigación futura puede considerar si otras mejoras siguen a la adopción de las NIIF plenas.

Varios estudios han documentado el proceso de adopción de las NIIF en los mercados en desarrollo. Por ejemplo, Jaruga et al., (2007) describen las dificultades de la conversión a las NIIF y el impacto de la adopción en los estados financieros en Polonia. Del mismo modo, Ionas et al., (2007) y Albu y Albu (2012) describen la naturaleza complicada del proceso en Rumania, mientras Sucher y Jindrichovska (2004) analizan la experiencia de la República Checa.

Algunos estudios proporcionan evidencia de los resultados económicos y contables de la adopción de las NIIF en los mercados emergentes de Europa Oriental. Dobija y Klimczak (2010) señalan que la eficiencia del mercado y la información relevante de valor se lograron temprano en el proceso de la contabilidad como un soporte de la privatización (desde 1997), pero estas características no mejoraron más en el periodo de 2005 a 2008 cuando se aplicaron las NIIF. Márquez-Ramos (2008) proporciona evidencia de que la adopción se asocia con más comercio e inversión extranjera directa y estos efectos positivos son más fuertes en estas economías en transición.

\section{Re-regulación financiera}

La regulación financiera ha emergido como una cuestión política importante tras la crisis económica de 2007-2008. En octubre de 2007, el Foro de Estabilidad Financiera (FEF) integró un grupo de trabajo para formular propuestas que optimizaran la estabilidad del mercado, la disciplina y la resistencia institucional a raíz de una crisis de liquidez en los mercados a partir de junio de ese mismo año (Helleiner y Pagliari, 2009).

Un plan de acción posterior de los ministros de Finanzas del G-20, sustentado en el trabajo del FEF, es descrito por Veron (2012) como de re-regulación: 
La realización renovada de los sistemas financieros, incluidos los sistemas bancarios, no puedan ser dejados a su propia suerte, tanto por los altos costos económicos potenciales de las crisis financieras y porque el gasto público es a menudo un componente clave para su resolución (p. 2).

El plan de acción de noviembre de 2008 del G-20 propuso un compromiso con los principios comunes para la reforma, que abarcaba el fortalecimiento de la transparencia y la rendición de cuentas, mejorar la regulación, promocionar la integridad de los mercados, reforzar la cooperación internacional, y la reforma de las instituciones financieras. Un conjunto de normas contables globales de aplicación y ejecución consistentes y mejores requisitos en relación con el reconocimiento, la medición y la revelación de los instrumentos financieros y entidades fuera del balance eran incluidos en los puntos de acción (Rottier y Veron, 2012). Subyacente a las iniciativas del G-20 se encontraba la idea de que las normas de contabilidad y la forma en que se aplicaban y se hacían cumplir debían ser mejoradas, dado su rol en los acontecimientos de la crisis financiera (Walker, 2010).

Los estudios han investigado el impacto de la NIC 39 Instrumentos Financieros: Reconocimiento y Medición sobre la contabilidad de instrumentos financieros siguiendo su adopción por los bancos de la UE a partir de 2005 (antes de los cambios relacionados con las propuestas del G-20). Leventis et al., (2011) estudiaron 91 entidades de la UE listadas en bolsa durante el periodo 1999-2008 e informan que la gestión de resultados (utilizando provisiones por pérdidas en préstamos) es más baja después de la adopción en 2005. Los autores concluyen que la aplicación de las NIIF en la UE ha mejorado la calidad de los resultados de los bancos mediante la mitigación de la tendencia de los gerentes de los bancos comerciales a participar de la gestión de resultados mediante provisiones por pérdidas en préstamos.

Gebhardt y Novotny-Farkas (2011) estudiaron 90 bancos de la UE en el periodo 2000-2007. Hallaron que la restricción de reconocer solo pérdidas incurridas de la NIC 39 redujo la estabilidad de los beneficios, aunque este resultado puede ser mitigado por la preferencia de los reguladores bancarios 
hacia provisiones por pérdidas en préstamos futuros. La aplicación de la aproximación de las pérdidas incurridas resultó en un reconocimiento menos oportuno de las provisiones por pérdidas en préstamos, lo que implicó igualmente el registro tardío de pérdidas esperadas futuras.

La respuesta del G-20 también hacía un llamado a la aplicación consistente de las normas contables. Antes de la crisis de 2007-2008, la Comisión Europea (CE) se mostró optimista con respecto a la calidad y la comparabilidad de los reportes bajo NIIF. Sobre la base de una serie de informes y exámenes de la información financiera ${ }^{5}$, un informe de la CE (2008) concluyó que no había una "percepción general entre los preparadores, auditores, inversores y supervisores de que la aplicación de las NIIF hubiera mejorado la comparabilidad y la calidad de la información financiera y haya dado lugar a una mayor transparencia” (p. 6).

Posteriormente, la Autoridad Europea de Valores y Mercados (ESMA, por su sigla en inglés) informó sobre las operaciones de supervisión en UE, y describió el nivel de actividad de varios organismos nacionales y su interacción en las European Enforcement Coordination Sessions (EECS) (ESMA, 2011). Los supervisores prestaron especial atención a la contabilidad de instrumentos financieros y encontraron mejoras significativas en 2009 y 2010 en el nivel de cumplimiento de los requisitos de revelación sobre técnicas de valoración, riesgo de crédito propio, riesgo de crédito, gananciaspérdidas y entidades de propósito especial. Sin embargo, concluyeron que algunas áreas (como la jerarquía de valor razonable y las revelaciones de riesgo cualitativos relacionados con los instrumentos financieros) requerían más atención por parte de los emisores.

Berger (2010) sentencia que las EECS han hecho una "contribución significativa" (p. 22) a la aplicación uniforme de las NIIF. Destaca que diferentes enfoques y métodos de enforcement se utilizan y varían ampliamente.

5 ICAEW (2007), CESR (2007) y reportes de la Accounting Regulatory Commission (ARC) de la Comisión Europea. 
Además, el grado en que los organismos publican sus actividades también cambia, lo que sugiere un área de mejora en el futuro.

En 2011, la SEC publicó una revisión de reportes bajo NIIF, sustentada en los estados financieros de 2009 de 183 sociedades cotizadas en los EE. UU que aplican las NIIF. El personal de la SEC encontró cumplimiento general de los requisitos de las NIIF, pero señaló que la transparencia y claridad de las revelaciones podría mejorarse. Además, concluyeron que "la diversidad en la aplicación representaba desafíos a la comparabilidad de los estados financieros entre países e industrias" (2011a, p. 2). El informe también menciona el uso de diferentes opciones en las NIIF, revelación insuficiente e inconsistente y la falta de orientación en algunas áreas como elementos con potencial para socavar la comparación.

\section{Las NIIF en los mercados de capitales desarrollados}

El llamado del G20 por un conjunto de normas comunes de contabilidad, para su uso en mercados de todo el mundo, plantea la pregunta sobre sus beneficios en los mercados que ya tienen altos niveles de transparencia y protección de los inversores. En el caso de Australia y Nueva Zelanda los responsables políticos piensan que el futuro crecimiento de sus pequeños mercados domésticos de capital requieren de inversión internacional, que sería promovida por el uso de las NIIF.

En Australia, la opinión fue apoyada de manera generalizada en las comunidades de negocios, aunque no necesariamente por todas las compañías individuales (Brown y Tarca, 2001). Algunas empresas más pequeñas consideraban que asumirían los costos de la adopción de las normas internacionales sin obtener los beneficios que pueden ser capitalizados por las corporaciones más grandes con los inversores y analistas internacionales (Jones y Higgins, 2006).

Los estudios sugieren que hay beneficios para el mercado de capitales tras la adopción de las NIIF, incluso en países con una fuerte protección a los 
inversores y con una información financiera de alta calidad y cumplimiento. Chalmers et al., (2011) reportaron un aumento en la relevancia del valor y la persistencia de los resultados cuando se adoptaron las NIIF en Australia.

Bissessur y Hodgson (2012) observaron una disminución de la synchonicity en el mercado de este país en los dos primeros años posteriores a la adopción de las NIIF (que llegó a ser mayor en el periodo 2007-2008). Bissessur y Hodgson (2012) y Cotter et al., (2012) encuentran más bajos errores de pronóstico post-NIIF en Australia, y Choi et al., (2010) reportan una situación similar para el Reino Unido.

Cheong et al., (2010) concluyen que la capitalización de intangibles según las NIIF proporciona información pertinente para determinar el valor a las empresas de Australia, Hong Kong y Nueva Zelanda. Bayerlein y Farooque (2012) reportan una mayor comparabilidad de las decisiones de política para impuesto diferido y plusvalía bajo las NIIF para las compañías de Australia, Reino Unido y Hong Kong. Brochet et al., (2011) también señalan mejor comparabilidad tras la adopción de las NIIF para las corporaciones del Reino Unido, tomando como referencia la propuesta de que la información privada se reduce después de dicha adopción.

Zeghal et al., (2011) informan que el uso de las NIIF se asocia con menos manipulación de resultados para las empresas francesas durante el periodo 2003-2006, sobre todo para aquellas con mayor gobierno corporativo y una mayor participación en los mercados financieros extranjeros.

Sun et al., (2011) examinan firmas de 23 países que cotizan en las bolsas de Estados Unidos. Exploran si la calidad de la contabilidad aumenta para estas empresas tras la adopción de las NIIF. Encuentran alguna evidencia de mejora, lo cual es sorprendente dado que cotizar en este país proporciona los incentivos para la presentación de informes de alta calidad antes del proceso de adopción. El estudio sugiere que las NIIF pueden tener un impacto en la contabilidad de las compañías en mercados desarrollados. 
También puede beneficiar a los inversionistas de un país, mediante la ampliación de su campo de inversiones potenciales. Lee y Farghar (2010) muestran que la adopción de las NIIF en Australia se asocia con un aumento en el nivel de inversión en títulos extranjeros por parte de inversionistas australianos.

Otra evidencia sobre los beneficios de las NIIF en los mercados desarrollados se refiere a la integración de los mercados de capital. Cai y Wong (2010) reportan una mayor correlación de los índices de mercado en países donde las empresas utilizan las NIIF (Reino Unido, Francia, Alemania e Italia) que en los mercados de naciones cuyas empresas locales no las suelen utilizar (Estados Unidos, Canadá, Japón y Rusia) para el periodo 1995-2008.

Sin embargo, no todos los estudios acerca de mercados desarrollados proporcionan evidencia de los beneficios de la adopción de las NIIF. Gjerde et al., (2008) en un estudio de las empresas en Noruega, hallan poca evidencia de un aumento general de la relevancia del valor de la información contable. Sin embargo, encuentran que la capitalización de los activos intangibles bajo las NIIF sí es relevante. Jarva y Lantto (2010) concluyen que las NIIF no proporcionan más información pertinente para determinar el valor y no permiten una mejor predicción de resultados o los flujos de efectivo futuros de las empresas en Finlandia.

En relación con otros mercados desarrollados, aún no hay suficiente evidencia disponible para Canadá, donde se aplican las NIIF desde 2011. Las razones de la adopción en este país son similares a las de otras jurisdicciones: el uso de las NIIF proporciona más oportunidades para las empresas e inversionistas canadienses al reducir el costo del capital, el aumento del acceso a los mercados internacionales y la reducción de costes al eliminar la necesidad de conciliaciones (CICA, 2012).

Lefebvre, de la Certified General Accountants Association (CGA) de Canadá, indicaba su acuerdo con la perspectiva de que las normas nacionales habían servido a Canadá cuando esta era una entidad económica autosuficiente, pero con la globalización el país debe aprovechar su capacidad de integración en el paisaje internacional (Jeffrey, 2011). 
En su informe anual para el periodo 2011-2012, el Canadian Accounting Standards Board (AcSB) reflexionó sobre la aplicación de las NIIF en Canadá. Si bien reconocía que algunas personas no estaban de acuerdo con la estrategia de adopción de las NIIF, concluía que no había nueva evidencia para llamar a las entidades y estados a cuestionarla y declaraba que "aunque las NIIF requieren mejoras, representan la única vía práctica para lograr el objetivo de un único conjunto de normas de información financiera de alta calidad, globalmente aceptado y que contribuya al mejor funcionamiento de los mercados de capital globales" (AcSB, 2012, p. 25).

Los argumentos sobre la participación en los mercados mundiales se aplican por igual en los Estados Unidos y Japón a pesar de que históricamente las economías de estos países se han encontrado entre las más grandes del mundo contemporáneo y se han servido de sus propias normas, reportes y estructuras de enforcement. Japón ha experimentado numerosas reformas en los estándares de regulación del mercado y de contabilidad financiera, sobre todo en la década de 1990, ya que el país ha buscado un papel relevante en el contexto financiero internacional (Misawa, 2005).

Saito (2008) argumenta que si Japón quiere un papel de liderazgo no puede aislarse de las normas internacionales de contabilidad. Aunque los PCGA de Estados Unidos han sido utilizados por algunas grandes empresas japonesas en el pasado, Saito (2008) afirma que estos no pueden ser el lenguaje global de la contabilidad, por lo que las normas contables japonesas deben armonizarse con la NIIF ${ }^{6}$. Desde 2005 el Consejo de Normas de Contabilidad de Japón (ASBJ, por sus siglas en inglés), ha llevado a cabo diversas iniciativas de armonización con el IASB, con el objetivo de lograr la convergencia internacional en las normas, así como contribuir a mercados de capitales más integrados. A pesar del tamaño e importancia de su mercado de capital, Japón ha experimentado la presión que se desprende

6 Saito (2008) no proporciona razones de por qué los PCGA de Estados Unidos no pueden ser las normas mundiales de contabilidad. Sin embargo, las posibles razones son su naturaleza basada en reglas y que hacen parte de un amplio conjunto de normas de presentación de informes, diseñado para un complejo marco regulatorio específico en los Estados Unidos. 
de que sus normas se consideren como "no equivalentes a las NIIF". La necesidad de reconocimiento de los PCGA de Japón en los mercados de la UE (véase Misawa, 2005) ha sido otro incentivo para la armonización de los PCGA nacionales y las NIIF y para que Japón adopte las NIIF.

Rottier y Veron (2010) notan que la geografía de las finanzas globales está cambiando. Los centros financieros asiáticos están aumentando en importancia y entre los 100 bancos más grandes del mundo, aproximadamente un tercio son de mercados en desarrollo. Estos autores argumentan que estas trasformaciones en los mercados financieros crean modificaciones de poder en el debate de política global. Por lo tanto, puede esperarse que Estados Unidos y Japón supervisen continuamente su función y participación en la regulación internacional de la información financiera y la actividad del mercado con el fin de mantener sus posiciones de influencia.

Antes de la crisis de 2007-2008, el aumento de la competencia en los mercados de capital dio lugar a llamamientos para que los Estados Unidos hiciesen cambios en su marco regulatorio a fin de renovar su eficiencia y al tiempo garantizar que el alto nivel de protección de los inversionistas se mantuviera (Cox y Greene, 2007; Pan, 2008). El supervisor estadounidense del mercado, la Securities and Exchange Commission (SEC), consideró varias propuestas de desregulación que disminuirían las barreras de entrada a los mercados estadounidenses. En 2008 eliminó el requisito de reconciliación PCGA de los Estados Unidos-NIIF para los extranjeros registrados en bolsa, y propuso una hoja de ruta que conduciría a la utilización de las NIIF por los usuarios domésticos en 2014 (Street, 2012). Las acciones respondían a la percepción de que los mercados de capitales estadounidenses estaban perdiendo su dominio anterior (Black, 2011) y se reconocía el uso generalizado y la aceptación de estados financieros bajo NIIF.

Las propuestas del G-20 llamaban a la acción de las entidades e instituciones tanto nacionales como internacionales, incluyendo algunas en Estados Unidos. Así, el Financial Accounting Standards Board (FASB) trabajó con el IASB en proyectos que trataban de normas contables sobre el reconocimiento, medición y revelación de los instrumentos financieros. 
Sin embargo, la posición de Estados Unidos sobre algunas cuestiones planteadas en las propuestas del G-20 (en concreto, un conjunto de normas globales) es menos favorable que en años anteriores. Por ejemplo, Street (2012) señala la decisión de la SEC de aplazar la entrada en vigencia de una recomendación a registrados estadounidenses en las bolsas locales para que usen las NIIF.

Aunque las condiciones económicas y políticas pueden haber cambiado después de 2008, los incentivos para que Estados Unidos sea un participante líder en el funcionamiento y la regulación de los mercados de capitales internacionales permanecen. Por ejemplo, Pan (2008) argumenta que la regulación bursátil de Estados Unidos dispone de desincentivos para las empresas, lo que lleva a menos emisores extranjeros a convertirse en sociedades públicas estadounidenses y a que junto con los inversionistas locales (y los proveedores de servicios financieros) muevan sus aumentos de capital y actividades de inversión fuera de los mercados del país. Jackson y Pan (2008) sugieren que la disminución de la competitividad estadounidense refleja más las tendencias constantes que una respuesta a la Ley Sarbanes Oxley o la Ley Federal de Valores.

Los eventos de la crisis de 2007-2008 no eliminaron los desincentivos identificados por Pan (2008). Así, quedan vigentes las motivaciones para que Estados Unidos tome partido en la convergencia, armonización y reconocimiento mutuo, a pesar de las dificultades que implica el proceso. La forma en que este y otros países gestionen la compensación entre la competencia y la cooperación se dará a conocer en el futuro. Los comentaristas señalan que mucho se espera de las instituciones globales en materia de regulación de los mercados de capital. Sin embargo, la participación de más países y significativamente menos la dominación de uno solo (o un bloque) hará que la toma de decisiones sea más difícil (Helliener y Pagliari, 2009). Más transparencia se exige, pero Rottier y Veron (2010) opinan que post 20072008 el grado de información comparable a nivel internacional sobre los sistemas financieros y los mercados, incluida la revelación sobre las finanzas públicas y el apoyo a las empresas financieras, sigue siendo insuficiente. 
La adopción de un conjunto de normas mundiales (NIIF), propuesto como uno de los elementos de los planes para mejorar la transparencia en los mercados financieros, parece tener menos apoyo en Estados Unidos y Japón desde la crisis de 2007-2008. Sin embargo, los investigadores sugieren que decisiones de adopción implican más que una consideración de los beneficios netos de las empresas nacionales. Chua y Taylor (2008) advierten contra la idea de atribuir la difusión de las NIIF únicamente a factores económicos, con el argumento de que los factores políticos y sociales tienen un papel importante. Como un ejemplo, apuntan a la circunstancia en la que "naciones fuertes" requieren a otros a adoptar las mejores prácticas internacionales, pero pueden ellas mismas no adoptar ciertas normas cuando les conviene.

Ramanna y Sletten (2009) concluyen que la adopción de las NIIF es más probable cuando los gobiernos son capaces de tomar decisiones pertinentes y cuando la oportunidad y los costos de cambio son relativamente bajos. Sin embargo, no encuentran que las decisiones de adopción estén relacionadas con el nivel de (o los cambios esperados en) el comercio exterior y los flujos de inversión. Por el contrario, sostienen que un país tiene más probabilidades de adoptar las NIIF cuando sus socios comerciales o vecinos regionales adoptan. Las percepciones de una red de beneficios, relacionados con costos de transacción menores para los usuarios extranjeros de los estados financieros, conducen a los países a tomar la decisión de adoptar las NIIF. Así, un país puede adoptar las NIIF, incluso si sus normas nacionales están particularmente bien adaptadas a sus instituciones nacionales (Ramanna y Sletten, 2011).

Las vacilaciones en los Estados Unidos sobre si, cómo y cuándo las NIIF podrían adoptarse son consistentes con la idea de un gobierno consciente de los costos de oportunidad del cambio. Durante la última década las partes constituyentes de Estados Unidos han estado expuestas a una gran variedad de estrategias para la incorporación o la adopción de las NIIF en el país (Erikson et al., 2009; Epstein, 2009). Curiosamente, un estudio basado en esta nación encuentra que los académicos y profesionales sostienen puntos 
de vista acerca de los beneficios y costos de las NIIF que son similares a los de sus homólogos de otras jurisdicciones. Rezaee et al., (2010) informaron de una encuesta realizada a 124 profesores y 120 profesionales (miembros del FEI ${ }^{7}$ en 2008 . Los primeros siete beneficios percibidos de la convergencia de los Estados Unidos con las NIIF se refieren a cuestiones globales (incluyendo la comparabilidad, uniformidad, inversión transfronteriza, integración, eficiencia y costo-efectividad de los mercados).

Aunque los gobiernos a menudo se preocupan por el tema de la soberanía (sobre normas contables como parte de la regulación de las empresas) y los costos de conversión para las empresas, los encuestados dieron también alta importancia a otros factores. Los asuntos de educación (falta de libros de texto basados en las NIIF y las Normas Internacionales de Auditoría; ausencia de enseñanza sobre las NIIF, incomprensión e inexperiencia de los elaboradores de la información) son relevantes. Otras preocupaciones se relacionan a la aplicación de las NIIF: uniformidad de la implementación y coordinación y colaboración entre los reguladores. Los profesionales clasificaban el costo de la conversión como el tercer obstáculo más importante mientras que los académicos lo situaban como el séptimo ${ }^{8}$.

\section{Conclusiones}

En este documento se ha revisado una serie de estudios que apuntan los beneficios de la adopción obligatoria de las NIIF. Usando una amplia variedad de técnicas de investigación, los trabajos demuestran que estas normas han mejorado la eficiencia de las operaciones del mercado de capitales y

7 El Financial Executives International (FEI) es una organización con sede en Estados Unidos e integrantes internacionales. El estudio no informó del número de encuestados educados o domiciliados en este país.

8 La encuesta no contiene referencia explícita a la "soberanía", pero las respuestas relativas a los "cambios necesarios en el régimen regulatorio" (preparadores sexto obstáculo más importante, académicos segundo) pueden referirse a estas preocupaciones. Sin embargo, no podemos estar seguros, dada la forma en que los datos se presentan y analizan. 
promovido la inversión transfronteriza. Además, muchos estudios señalan la importancia de la infraestructura que rodea el uso de las NIIF.

Hegarty et al., (2004) afirman que una "combinación completa y equilibrada de la capacidad y los incentivos institucionales" para la aplicación rigurosa de las normas internacionales de contabilidad y auditoría es la clave del éxito de su implementación. La evidencia de las investigaciones muestra sistemáticamente que los beneficios de las NIIF son más propensos a hacerse realidad cuando su aplicación se apoya en un marco que abarca las protecciones legales, profesionales competentes y un monitoreo y enforcement adecuados.

Los beneficios potenciales de las NIIF en los mercados en vías de desarollo pueden ser fuertemente argumentados. Los llamados por las ventajas del uso de las NIIF en los mercados desarrollados son más polifacéticos. Entre los Estados miembros de la UE, los países han adoptado obligatoriamente las NIIF porque desean buenos resultados para el grupo en su conjunto. Sin embargo, la magnitud del cambio para un país en particular y por lo tanto el beneficio para las empresas a nivel nacional varía entre los integrantes de la eurozona.

No es sorprendente que muchos estudios apunten a la variación de los beneficios de las NIIF, cuando se examinan país por país (Brown, 2011; Pope y McLeay, 2011; Brüggemann et al., 2012). Si las ventajas de las NIIF están ligadas a factores tales como las diferencias entre estas y los PCGA nacionales y el grado de aplicación legal, como se explicó antes, entonces deberíamos esperar a ver divergencias en los beneficios a nivel de cada país. Sin embargo, un cuerpo emergente de investigación apunta a los beneficios globales del uso de las NIIF, en particular cuando la implementación está acompañada por una buena infraestructura. 


\section{Referencias}

Albu, N., \& Albu, C. (2012). IFRS in an emerging economy: Lessons from Romania. Australian accounting review, 22(4), 341-352.

Bae, K. H., Tan, H., \& Welker, M. (2008). International GAAP differences: The impact on foreign analysts. Accounting review, 83(3), 593-628.

Ball, R., A. Robin, \& Wu, J. (2003). Incentives versus standards: properties of accounting income in four East Asian countries and implications for the acceptance of IAS Journal of Accounting and Economics, 36, 235-270.

Ball, R., \& Brown, P. (1968). An empirical evaluation of accounting income numbers. Journal of accounting research, 6(2), 159-178.

Ball, R. S., \& Robin, A. (2000). The effect of international institutional factors on properties of accounting earnings. Journal of accounting and economics, 29(1), 1-51.

Bayerlein, L., \& Farooque, O. (2012). Influence of a mandatory IFRS adoption on accounting practice: Evidence from Australia, Hong Kong and the United Kingdom. Asian review of accounting, 20(2), 93-118.

Beaver, W. H. (1968). The information content of annual earnings announcements. Journal of accounting research, 6, 67-92.

Beneish, M., Miller, B., \& Yohn, T. (2010). The effect of IFRS on adoption on cross-border investment in equity and debt markets. Working paper. Indiana University.

Berger, A. (2010). The development and status of enforcement in the European Union. Accounting in Europe, 7(1), 15-35.

Beuselinck, C., Joos, P., Khurana, I., \& Meulen, S. V. (2010). Mandatory adoption of IFRS and analysts' forecasts information properties. SSRN eLibrary.

Beuselinck, C., Joos, P., Khurana, I., \& Van der Meulen, S. (2009). Mandatory IFRS reporting and stock price informativeness. SSRN eLibrary.

Bissessur, S., \& Hodgson, A. (2012). Stock market synchronicity - an alternative approach to assessing the information impact of Australian IFRS. Accounting \& finance, 52(1), 187-212.

Black, B. (2011). Introduction: The globalization of securities regulation - competition or coordination? University of Cincinnati law review, 79(2). 
Brochet, F., Jagolinzer, A. D., \& Riedl, E. J. (2011). Mandatory IFRS adoption and financial statement comparability. SSRN eLibrary.

Brown, P. (2011). International financial reporting standards: How real are the benefits? Accounting and business research, 41(3), 269-285.

Brown, P., \& Tarca, A. (2001). Politics, processes and the future of Australian accounting standards. Abacus, 37(3), 267-296.

Brüggemann, U., Daske, H., Homburg, C., \& Pope, P. F. (2009). How do individual investors react to global IFRS adoption? SSRN eLibrary.

Bushman, R., Piotroski, J., \& Smith, A. (2004). What determines corporate transparency? Journal of accounting research, 42(2), 207-251.

Byard, D., Li, Y., \& Yu, Y. (2011). The effect of mandatory IFRS adoption on financial analysts' information environment. Journal of accounting research, 49(1), 69-96.

Cai, F., \& Wong, H. (2010). The effect of IFRS adoption on global market integration. International business and economics research journal, 9(10), 25-34.

Chalmers, K., Clinch, G., \& Godfrey, J. M. (2011). Changes in value relevance of accounting information upon IFRS adoption: Evidence from Australia. Australian journal of management, 36(2), 151-173.

Chen, C., Ding, Y., \& Xu, B. (2011). Convergence of accounting standards and foreign direct investment. Working paper. City University of Hong Kong and China Europe International Business School.

Cheong, C. S., Kim, S., \& Zurbruegg, R. (2010). The impact of IFRS on financial analysts' forecast accuracy in the Asia-Pacific region: The case of Australia, Hong Kong and New Zealand. Pacific accounting review, 22(2), 124-146.

Christensen, H. B., Lee, E., \& Walker, M. (2008). Incentives or standards: What determines accounting quality changes around IFRS Adoption? SSRN eLibrary.

Christensen, H. B., Hail, L., \& Leuz, C. (2010). Capital-market effects of securities regulation: The role of implementation and enforcement. SSRN eLibrary.

Christensen, H. B., Hail, L., \& Leuz, C. (2012). Mandatory IFRS reporting and changes in enforcement. SSRN eLibrary.

Chua, W. F., \& Taylor, S. L. (2008). The rise and rise of IFRS: An examination of IFRS diffusion. Journal of accounting and public policy, 27(6), 462-473.

CICA. (2012). IFRS frequently asked questions. Chartered accountants of Canada. 
Cotter, J., Tarca, A., \& Wee, M. (2012). IFRS adoption and analysts' earnings forecasts: Australian evidence. Accounting and finance, 52(2), 395-419.

Cox, J., \& Greene, E. (2007). Financial regulation in a global market place: Report of the Duke global capital market roundtable. 239-252.

Daske, H., Hail, L., Leuz, C., \& Verdi, R. S. (2008). Mandatory IFRS reporting around the world: Early evidence on the economic consequences. Journal of accounting research, 46(5), 1.085-1.142.

Daske, H., Hail, L., Leuz, C., \& Verdi, R. S. (2011). Adopting a Label: Heterogeneity in the economic consequences of IFRS adoptions. SSRN eLibrary.

DeFond, M., Hu, X., Hung, M., \& Li, S. (2011). The impact of mandatory IFRS adoption on foreign mutual fund ownership: The role of comparability. Journal of accounting and economics, 51(3), 240-258.

Dobija, D., \& Klimczak, K. M. (2010). Development of accounting in Poland: Market efficiency and the value relevance of reported earnings. The international journal of accounting, 45(3), 356-374.

Epstein, B. (2009). The economic effects of IFRS adoption. The CPA Journal (march), 26-31.

Erickson, D., Esplin, A., \& Maines, L. A. (2009). One world - One accounting. Business horizons, 52(6), 531-537.

Ernstberger, J., Krotter, S., \& Stadler, C. (2008). Analysts' forecast accuracy in Germany: The effect of different accounting principles and changes of accounting principles. BuR-Business research journal, 1(1), 26-53.

European Securities and Markets Authority (ESMA). (2011). Activity report on IFRS enforcement in 2010. Brussels.

Fifield, A. (2007). Korea moves towards full adoption of IFRS. Financial Times.

Florou, A., \& Pope, P. F. (2012). Mandatory IFRS adoption and investor asset allocation decisions. SSRN eLibrary.

Francis, J., Huang, S. \& Khurana, 1. (2012). The role of international GAAP in cross-border mergers and acquisitions. Working paper. University of Arkansas.

Frost, C., Gordon, E., \& Hayes, A. (2001). Stock exchange disclosure and market liquidity: An analysis of 50 international exchanges. Journal of accounting research, 44(3), 437-483.

Gebhardt, G., \& Novotny-Farkas, Z. (2011). Mandatory IFRS adoption and accounting quality of European banks. Journal of business finance \& accounting, 38(3-4), 289-333. 
Gjerde, O., Knivsflå, K. H., \& Sættem, F. (2008). The value-relevance of adopting IFRS: Evidence from 145 NGAAP restatements. SSRN eLibrary.

Gordon, L. A., Loeb, M. P., \& Zhu, W. (2012). The impact of IFRS adoption on foreign direct investment. Journal of accounting and public policy, 31(4), 374-398.

Hegarty, J., Gielen, F., \& Barros, A. (2004). The implementation of international accounting and auditing standards: Lessons learned from the world bank's accounting and auditing ROSC program: The world bank.

Helleiner, E., \& Pagliari, S. (2009). Towards a new Bretton Woods? The first G20 leaders summit and the regulation of global finance. New political economy, 14(2), 275-287.

Hodgdson, C., Tondkar, R. H., Harless, D. W., \& Adhikari, A. (2008). Compliance with IFRS disclosure requirements and individual analysts' forecast errors. Journal of international accounting, auditing and taxation, 17(1), 1-13.

Hope, O.-K., Jin, Y. J., \& Kang, T. (2006). Empirical evidence on jurisdictions that adopt IFRS. SSRN eLibrary.

Horton, J., Serafeim, G., \& Serafeim, I. (2013). Does mandatory IFRS adoption improve the information environment? Contemporary accounting research, $30(1), 388-423$.

IASPLUS. (2012, 12 september). AcSB reflects on Canadian IFRS adoption strategy. Available in www.iasplus.com

Ionas, I., Ionas, M., Olimid, L., \& Artemisa, D. (2007). An empirical evaluation of the costs of harmonizing Romanian accounting with international regulations (EU Directives and IAS/IFRS). Accounting in Europe, 4(2), 169-206.

Jackson, H. E., \& Pan, E. J. (2008). Regulatory competition in international securities markets: Evidence from Europe part II. Virginia law and business review, 3(2), 207-273.

Jackson, H. E., \& Roe, M. J. (2009). Public and private enforcement of securities laws: Resource-based evidence. SSRN eLibrary.

Jaruga, A., Fijalkowska, J., Jaruga-Baranowska, M., \& Frendzel, M. (2007). The impact of IAS/IFRS on Polish accounting regulations and their practical implementation in Poland. Accounting in Europe, 4(1), 67-78.

Jarva, H., \& Lantto, A. M. (2010). The value-relevance of IFRS versus domestic accounting standards: Evidence from Finland. SSRN eLibrary.

Jeffrey, G. (2007). The end of Canadian GAAP? Canada: CGA. 
Jiao, T., Koning, M., Mertens, G., \& Roosenboom, P. (2012). Mandatory IFRS adoption and its impact on analysts' forecasts. International review of financial analysis, 21, 56-63.

Jones, S., \& Higgins, A. D. (2006). Australia’s switch to International Financial Reporting Standards: A perspective from account preparers. Accounting and finance, 46(4), 629-652.

Kadri, M. H., \& Zulkifli, M. (2008). Relationship between market value and book value of Malaysian firms under pre and post FRS. SSRN eLibrary.

Kvaal, E., \& Nobes, C. (2011). IFRS Policy Changes and the Continuation of National Patterns of IFRS Practice. European accounting review, 21(2), 343-371.

Kvaal, E., \& Nobes, C. W. (2010). International differences in IFRS policy choice. Accounting and business research, 40(2), 173-187.

La Porta, R., F. Lopez-de-Silanes, Shleifer, A., \& Vishny, R. (1997). Legal determinants of external finance. Journal of finance, 52(3), 1.131-1.150.

La Porta, R., Lopez-de-Silanes, F., Shleifer, A., \& Vishny, R. (1998). Law and finance. Journal of political economy, 106(6), 1.113.

Landsman, W. R., Maydew, E. L., \& Thornock, J. R. (2011). The information content of annual earnings announcements and mandatory adoption of IFRS. Journal of accounting and economics, 53(1-2), 34-54.

Lang, M. H., \& Lundholm, R. J. (1996). Corporate disclosure policy and analyst behavior. The accounting review, 71(4), 467-492.

Lang, M. H., Maffett, M. G., \& Owens , E. L. (2010). Earnings comovement and accounting comparability: The effects of mandatory IFRS adoption. SSRN eLibrary.

Lee, G., \& Fargher, N. (2010). Did the adoption of IFRS encourage cross-border investment? Working paper. Australian National University.

Leuz, C., Nanda, D., \& Wysocki, P. (2003). Earnings management and institutional factors: An international comparison. Journal of financial economics, 69(3), 505-527.

Leventis, S., Dimitropoulos, P., \& Anandarajan, A. (2011). Loan loss provisions, earnings management and capital management under IFRS: The case of EU commercial banks. Journal of financial services research, 40(1), 103-122.

Li, S. (2010). Does mandatory adoption of International Financial Reporting Standards in the European Union reduce the cost of equity capital? The accounting review, 85(2), 607-636. 
Malaysian Accounting Standards Board (MASB). (2008). Malaysia's Convergence with IFRS in 2012. Available in http://www.masb.org.my

Marques-Ramos, L. (2011). European accounting harmonization: Consequences of IFRS and adoption on trade in goods and foreign direct investments. Emerging markets finance and trade, 47(5), 42-57.

Misawa, M. (2005). The Japanese issues and perspectives on convergence of accounting standards. Northwestern journal of international law and business, 25(3), 711-745.

Palea, V. (2007). The effects of the IAS/IFRS adoption in the European Union on the financial industry. The European Union review, 12(1-2).

Pan, E. (2008). The new internationalization of US securities regulation: Improving the prospects for a trans-Atlantic marketplace. European company law, 5(2), 73-78.

Pan, E. J., \& Jackson, H. E. (2008). Regulatory competition in international securities markets: Evidence from Europe - part II. Virginia law and business review, 3(2), 208-273.

Panaretou, A., Shackleton, M. B., \& Taylor, P. A. (2013). Corporate risk management and hedge accounting. Contemporary accounting research, 30(1), 116-139.

Pope, P., \& McLeay, S. (2011) The European IFRS experiment: Objectives, research challenges and some early evidence. Accounting and business research 41(3), 233-266.

Ramanna, K., \& Sletten, E. (2009). Why do countries adopt International Financial Reporting Standards? SSRN eLibrary.

Ramanna, K., \& Sletten, E. (2011). Network effects in countries adoption of IFRS. SSRN eLibrary.

Rezaee, Z., Smith, L. M., \& Szendi, J. Z. (2010). Convergence in accounting standards: Insights from academicians and practitioners. Advances in accounting, 26(1), 142-154.

Rottier, S., \& Veron, N. (2010a). An assessment of the G20's initial action items, 8, 1-13. Bruegel Policy Contribution.

Rottier, S., \& Veron, N. (2010b). Not all financial regulation is global, (10)22, 1-9. Washington, DC.

Saito, S. (2008). Significance of convergence and the role of IFRS in Japan: To encourage IFRS to be accepted by converging countries. Working paper. Meiji Gakuin University Japan. 
Shima, K. M., \& Gordon, E. A. (2011). IFRS and the regulatory environment: The case of U.S. investor allocation choice. Journal of accounting and public policy, 30(5), 481-500.

Street, D. (2012). IFRS in the US: If, when and how. Australian accounting review, 22(3), 257-274.

Sucher, P., \& Jindrichovska, I. (2004). Implementing IFRS: A case study of the Czech Republic. Accounting in Europe, 1(1), 109-141.

Tan, H., Wang, S., \& Welker, M. (2011). Analyst following and forecast accuracy after mandated IFRS adoptions. Journal of accounting research, 49(5), 1.307-1.357.

Veron, N. (2012). Financial reform after the crises: An early assessment, 8, 1-15. Bruegel working paper.

World Bank. (2011). Reports on the Observance of Standards and Codes (ROSC). Available in http://www.worldbank.org

Walker, M. (2010). Accounting for varieties of capitalism: The case against a single set of global accounting standards. British accounting review, 42(3), 137-152.

Wan Ismail, W. A., Dunstan, K. L., \& Van Zijl, T. (2010). Earnings quality and the adoption of IFRS-based accounting standards: Evidence from an emerging market. SSRN eLibrary.

Wang, J., \& Yu, W. (2009). The information content of stock prices, reporting incentives and accounting standards: International evidence. SSRN eLibrary.

$\mathrm{Yu}, \mathrm{G}$. (2009). Accounting standards and international portfolio holdings: Analysis of cross-border holdings following mandatory adoption of IFRS. SSRN eLibrary.

Zéghal, D., Chtourou, S., \& Sellami, Y. M. (2011). An analysis of the effect of mandatory adoption of IAS/IFRS on earnings management. Journal of international accounting, auditing and taxation, 20(2), 61-72. 